\title{
Direct measurement of the absolute light refractive index of air with the made double Michelson interferometer
}

Jozef Babiak

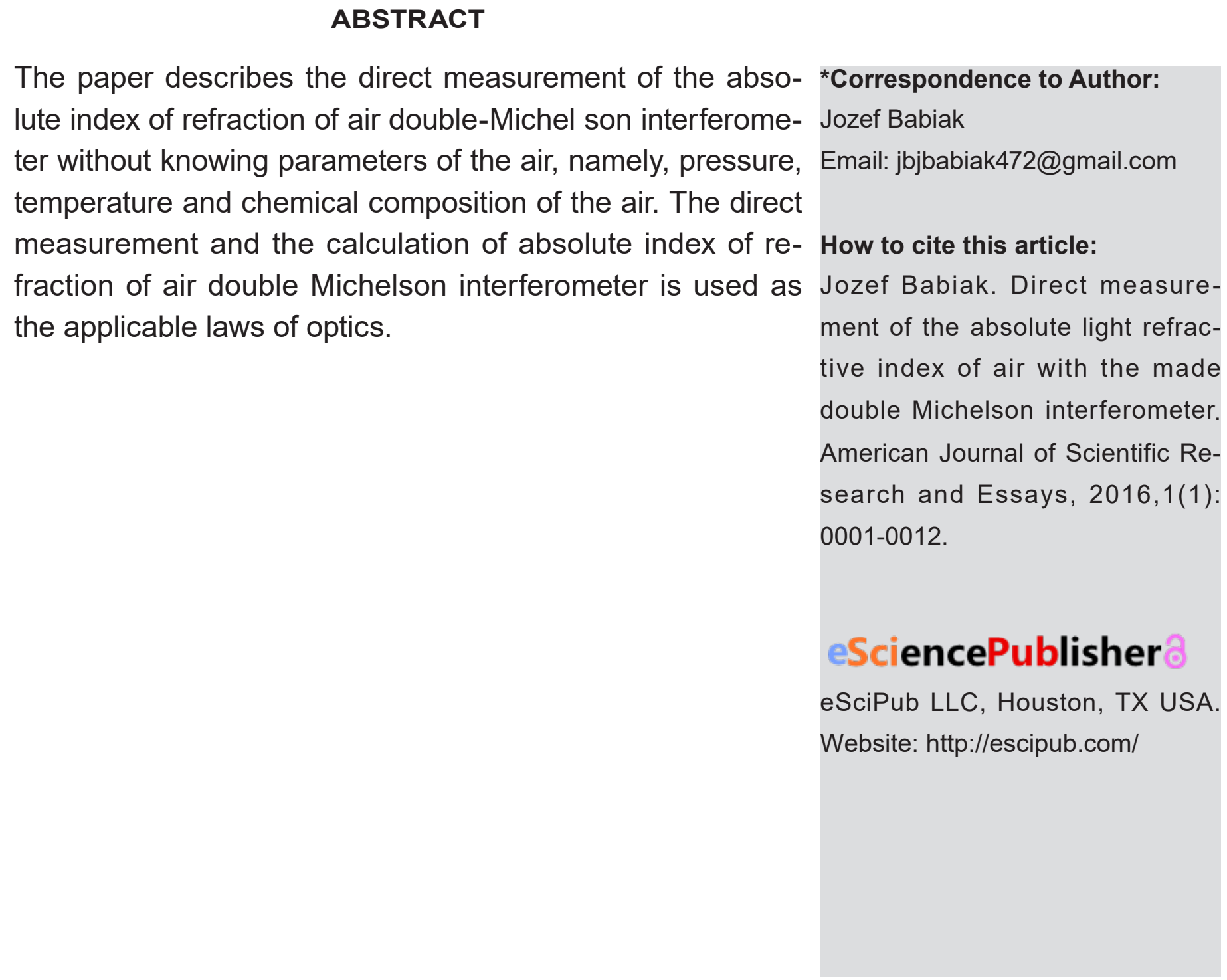




\section{Introduction}

The absolute light refractive index of a material is a dimensionless physical quantity and is defined as the ratio of the speed of light in vacuum c and the speed of light in the medium. It can be expressed by the equation $\mathbf{n}=\mathbf{c} / \mathbf{u}$. In optics, the absolute light refractive index is described as the second law of optics [1]. The absolute light refractive index is a very important value in the calculation of the trajectory of a light ray in optical instruments and topography practice for the calculation of the measured length in the field. The precise measurement of the absolute light refractive index in the atmosphere was carried out by a Fabry-Pérot resonator. The measurement is described in article [2]. Edlén formulated from the measured results the Equation for the Refractive Index of Air for the frequency of light, pressure, temperature, humidity and chemical composition of the air. The same equations for the absolute light refractive index were also formulated by Sellmeier as referred to in article [3]. From Edlén and Sellmeier's equations, we can calculate the absolute light refractive index by inserting the measured values of pressure, temperature, humidity and chemical composition of the air. This measurement is indirect, because it does not measure the absolute light refractive index in air. The air in the atmosphere contains many gases and gas compounds in different concentrations and some gases have a great impact on the value of the absolute light refractive index of air. Edlén and Sellmeier's equations do not apply to air with an unknown concentration and composition of gases, because they only take into account the influence of some gases and their compositions in air.

The absolute light refractive index of air in the atmosphere is a very important value in topography practice for the precise calculation of the length measured by electronic distance measuring devices. An electronic distance measuring device measures the time in which a ray of light passes along the trajectory, measured in the air, at the speed of light in air using the radar principle. The length of the measured trajectory in the air equals the conjunction of the time measured by the electronic distance measuring device, multiplied by the speed of light in air on the measured trajectory. The speed of light in air can be calculated with the help of the absolute refractive index of light in the atmosphere. The measurements of the absolute light refractive index in air made by Edlén and Sellmaier showed that the absolute light refractive index as well as the speed of light in the air depends on the frequency of the light source in a vacuum and on the parameters of the air in which the light is propagated. The article describes the new principle of direct measurement abolútného refractive index of air double Michelson interferometer without airflow through the measured parameters measured shifts of interference fringes in the two interferometer. The measurement and the calculation of absolute index of refraction of air double Michelson interferometer is used only applicable laws of optics. The speed of light and the wavelength of light is referred to by the frequency of light and air parameters at the point of light. The first index indicates the speed of light by the frequency of light, the first index " $h$ " denotes a helium laser light. The second subscript "a" denotes the parameters of the atmosphere air in the interferometer arms. New labeling parameters of light by frequency and parameters of the air at the end of the article.

A new indication of the speed and wavelength of light in the air of the surrounding atmosphere valid for the light of a helium laser can be seen in the following equation:

$$
u_{b}=\frac{c_{h}}{n_{h}}=v_{h} \lambda_{h}=v_{h} \frac{\lambda_{h}}{n_{h}}=n_{b} v_{h} \frac{\lambda_{h}}{n_{h}}
$$

It results from the equation that the light entering the second matter changes the speed of light, wavelength and frequency of the light in the second matter. I verified the validity of the equation by measuring the absolute light refractive index in air with the help of a double Michelson interferometer, see measurement results in a Graph 1.

\section{New principle for the direct measurement of the absolute light refractive index in air by using the interferometer} I proposed and carried out a new principle for the direct measurement of the absolute light refractive index in air with the help of a modified double Michelson interferometer as referred to in Figure 9. The principle of the direct measurement of the absolute light refractive index is based on 


\section{Measurement of the absolute index of refraction Michelsoninterferometer}

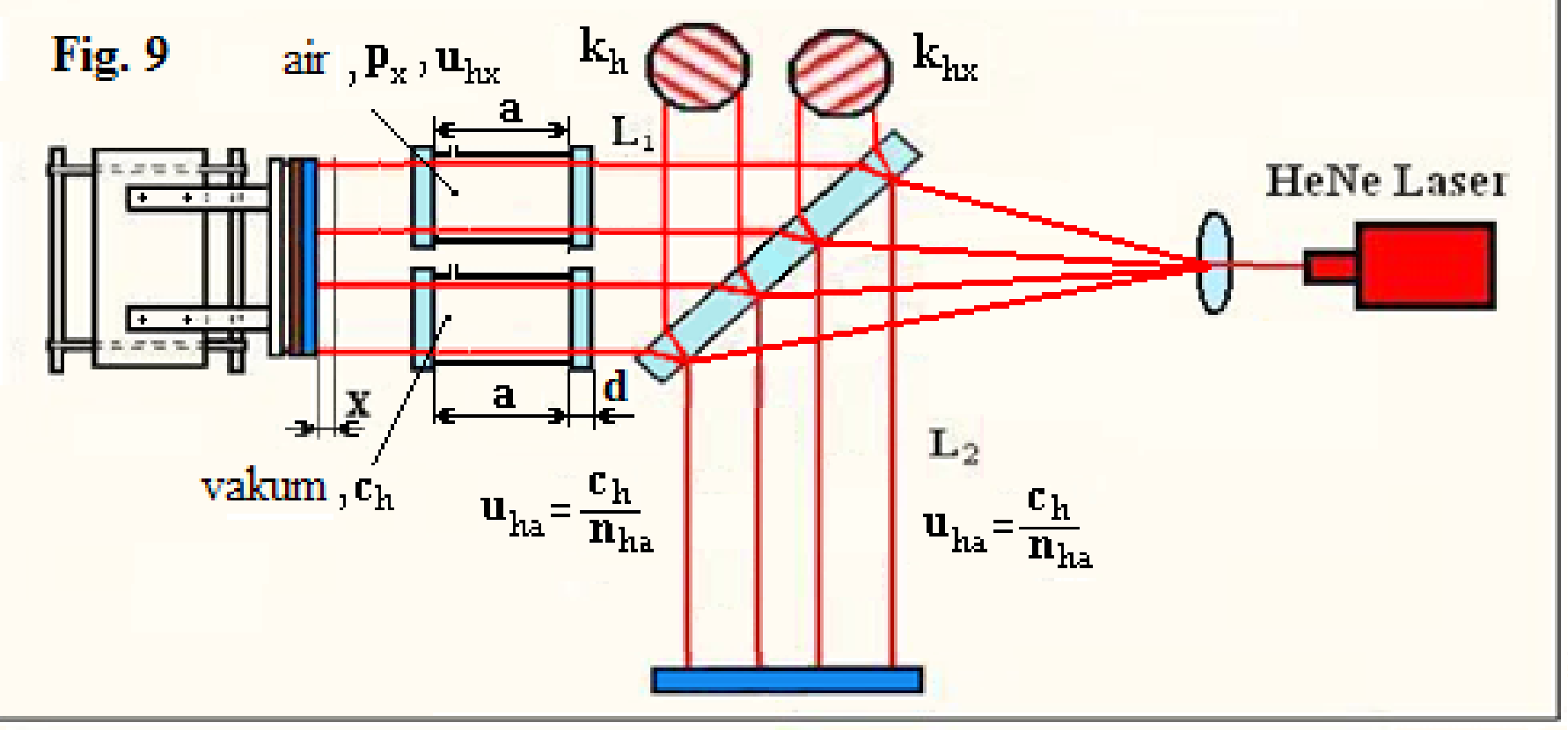

the principle of comparison between the speed of light in air and the speed of light in a vacuum.

The double Michelson interferometer for the direct measurement of the absolute light refractive index in the air of the surrounding atmosphere is made up of two Michelson interferometers positioned in parallel to each other. By inserting two pressure pipes into the ray of light in one arm of the interferometer, we obtain two interferometers with two ground glasses. The first interferometer is made by inserting the pressure pipe into one arm of the interferometer, where it acts as a vacuum. The second interferometer is made by inserting the pressure pipe in which the air of the surrounding atmosphere is measured.

The direct measurement of the absolute light refractive index of air present in the pressure pipe can be carried out by a mirror shift in air in one arm of the interferometer. The continuous shift of the interferometer mirror is carried out by shifting the platform on which the mirrors are placed with the help of a threaded rod rotated by an electric motor. The speed of the mirror shift is 3 to 5 wavelengths of light per second for the electronic reading of shifts of interference fringes on the ground glasses of the interferometer. The speed of the platform shift can be reduced by reducing the turns of the threaded rod using a gearbox with the high torque gearbox of an electric motor. The continuous shift of the mirror will cause a continuous shift in interference fringes on the ground glasses of both interferometers without shaking the interference fringes. The shift of the mirror in one arm of both interferometers on the length $\mathbf{x}$ in the surrounding atmosphere causes a frequency different shift in interference fringes on the ground glasses of both interferometers. The different shift of the interference fringes on the ground glasses of both interferometers is caused by the difference between the speed of light in the air in the pressure pipe and the speed of light in the pressure pipe in the vacuum. The shift of interference fringes on the ground glasses of both interferometers is electronically registered as electrical voltage on mini phototransistors. The change in the brightness of the light is caused by the shift of interference fringes on the ground glasses of the interferometers. The change in the brightness of the light on the phototransistor invokes a change in electrical voltage on the phototransistor, which is converted by AC converters into digital values which are entered into the computer memory for the numeric calculation of the absolute light refractive index in air. The shift of the interference fringes on the ground glass of the interferometer with the pipe and the air of the surrounding atmosphere and the shift of the interference fringes on the ground glass of interferometer with the pipe with a vacuum enables us to calculate the absolute light refractive index of the surrounding atmosphere in pressure pipe. Mathematical calculation absolute index 


\section{Measurement of the absolute index of refracion of air move in vacuo and}

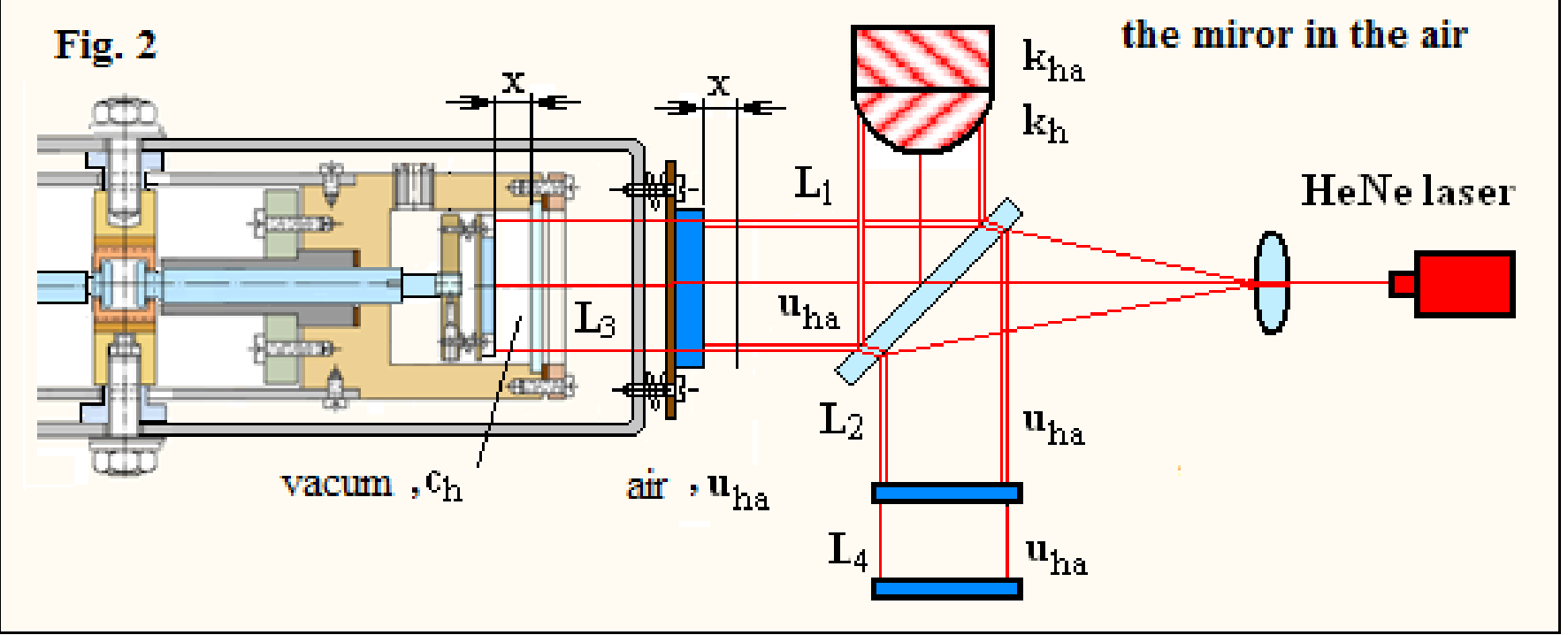

of refraction of air interferometer from the measured shifts of interference fringes $\mathbf{k}_{h}$, $\mathbf{k}_{\text {ha }}$ is calculated from the speed of light in vacuum and in air according to Fig. $\mathbf{9}$. The equation for the shift of interference fringes on ground glass $\mathbf{k}_{\mathrm{h}}$

$$
\begin{aligned}
& t_{1} u_{h a}=x+L_{1}-a-2 d, t_{2} u_{h s}=2 d, t_{3} c_{h}=a, t_{4} u_{h a}=L_{2} \\
& u_{h a}\left(2 t_{1}+2 t_{2}+2 t_{3}-2 t_{4}\right)=k_{h} \lambda_{h a}, 2 u_{h a}\left(\frac{x+L_{1}-a-2 d}{u_{h a}}+\frac{a}{c_{h}}+\frac{2 d}{u_{h s}}-\frac{L_{2}}{u_{h a}}-\right)=k_{h} \lambda_{h a} \\
& 2 x+2 L_{1}-2 L_{2}+2 a\left(\frac{u_{h a}}{c_{h}}-1\right)+4 d\left(\frac{u_{h a}}{u_{h s}}-1\right)=k_{h} \lambda_{h a}
\end{aligned}
$$

The equation for the shift on interference fringes on ground glass $\mathbf{k}_{\mathrm{hx}}$ :

$$
\begin{aligned}
& t_{1} u_{h a}=x+L_{1}-a-2 d, t_{2} u_{h s}=2 d, t_{3} u_{h x}=a, t_{4} u_{h a}=L_{2} \\
& u_{h a}\left(2 t_{1}+2 t_{2}+2 t_{3}-2 t_{4}\right)=k_{h x} \lambda_{h a}, 2 u_{h a}\left(\frac{x+L_{1}-a-2 d}{u_{h a}}+\frac{a}{u_{h x}}+\frac{2 d}{u_{h s}}-\frac{L_{2}}{u_{h a}}-\right)=k_{h x} \lambda_{h a} \\
& 2 x+2 L_{1}-2 L_{2}+2 a\left(\frac{u_{h a}}{u_{h x}}-1\right)+4 d\left(\frac{u_{h a}}{u_{h s}}-1\right)=k_{h x} \lambda_{h a}
\end{aligned}
$$

Subtracting one equation from another we come to following result:

$$
\begin{aligned}
& \mathrm{k}_{\mathrm{h}} \lambda_{\mathrm{ha}}-\mathrm{k}_{\mathrm{h} \mathrm{\lambda}} \lambda_{\mathrm{ha}}=2 \mathrm{a}\left(\frac{\mathrm{u}_{\mathrm{ha}}}{\mathrm{c}_{\mathrm{a}}}-\frac{\mathrm{u}_{\mathrm{ha}}}{\mathrm{u}_{\mathrm{hx}}}\right)=2 \mathrm{a}\left(\frac{1}{\mathrm{n}_{\mathrm{ha}}}-\frac{\mathbf{n}_{\mathrm{hx}}}{\mathbf{n}_{\mathrm{ha}}}\right)=\frac{2 \mathrm{a}}{\mathrm{n}_{\mathrm{ha}}}\left(1-\mathrm{n}_{\mathrm{hx}}\right),\left(\mathrm{k}_{\mathrm{h}}-\mathrm{k}_{\mathrm{hx}}\right) \lambda_{\mathrm{h}}=2 \mathrm{a}\left(1-\mathbf{n}_{\mathrm{hx}}\right) \\
& \mathrm{n}_{\mathrm{hx}}=1+\left(\mathrm{k}_{\mathrm{hx}}-\mathrm{k}_{\mathrm{h}}\right) \frac{\lambda_{\mathrm{h}}}{2 \mathrm{a}}
\end{aligned}
$$

The new principle for the direct measurement of the absolute light refractive index in air by using a Michelson interferometer does not require knowledge of the measured parameters of the air in the pressure pipe as pressure, temperature and composition of the air. The double Michelson interferometer measures directly the absolute light refractive index in the air present in the pressure pipe from the different shifts of interference fringes on the ground glasses of both interferometers caused by the different speeds of light in the air and in the vacuum!

A comparison of the wavelength of laser light in air to the wavelength of the laser light in vacuum. Comparison wavelengths of light in the air helium laser with a wavelength of laser helium vacuum double Michelson interferometer measures of Fig. 2. The double Michelson interferometer for measuring is made of a pressure pipe with a mirror shift in a vacuum and a second mirror with the shift in air of the surrounding atmosphere. The second interferometer can be obtained by inserting the mirror in the air in the upper half of the ray of light of the HeNe laser in one arm of the interferometer. The shift of both mirrors in the interferometers is obtained by the mechanical connection of mirrors and by rotating the threaded rod with an electric motor. The lower half of the ray of light impinges on the shifting mirror in the pipe with a vacuum. The upper half of the rays of light impinges on a mirror in the surrounding atmosphere. Both mirrors are connected and shifted on the same trajectory $\mathbf{x}$ in the air and in the vacuum.

The shift of the mirror in the vacuum by length $\mathbf{x}$ is equal to the shift of the mirror in the air in the surrounding atmosphere. The equations of the interferometer for the mirror shift can be written directly from Figure 2 . $\mathbf{x}=\mathbf{k}_{\mathrm{h}} \lambda_{\mathrm{h}}=\mathbf{k}_{\mathrm{ha}} \lambda_{\mathrm{ha}}=\mathbf{k}_{\mathrm{ha}} \frac{\lambda_{\mathrm{h}}}{\mathbf{n}_{\mathrm{ha}}}, \mathbf{k}_{\mathrm{h}} \lambda_{\mathrm{h}}=\mathbf{k}_{\mathrm{ha}} \frac{\lambda_{\mathrm{h}}}{\mathbf{n}_{\mathrm{ha}}}, \quad \mathbf{n}_{\mathrm{ha}}=\frac{\mathbf{k}_{\mathrm{ha}}}{\mathbf{k}_{\mathrm{h}}}$ 


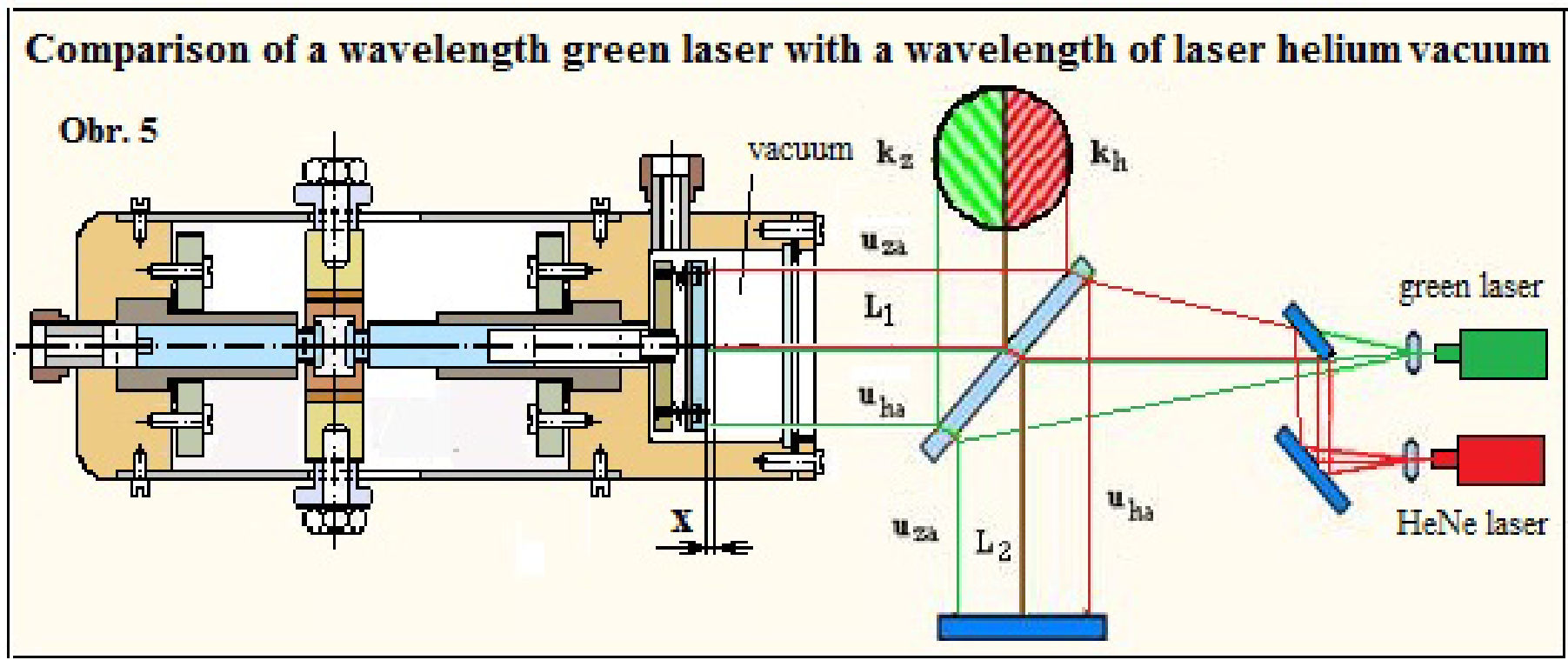

Comparison of a wavelength green laser with a wavelength of laser helium vacuum Comparison wavelength green laser light with a wavelength of a helium laser interferometer dual vacuum tube in Fig. 5. Double interferometer is enlightened by a helium laser on the upper half mirror, mirror on the bottom half of the enlightened green laser. Of the displaced interference fringes $\mathbf{k}_{\mathrm{h}}, \mathbf{k}_{\mathrm{ha}}$ caused a shift of the mirror in a vacuum in one arm of the interferometer calculate wavelength green laser vacuum $\lambda_{z}$. On the bottom half of the sliding mirror vacuum in the pipe turns green laser light. On the top half of the sliding mirror vacuum in the tube light it falls helium laser mirror is moved on the same track $x$ vacuum. Withthis, we can directlywritetheequation

$$
\mathbf{x}=\mathbf{k}_{2} \lambda_{\mathrm{z}}=\mathbf{k}_{\mathrm{h}} \lambda_{\mathrm{h}}, \lambda_{\mathrm{z}}=\lambda_{\mathbf{h}} \frac{\mathbf{k}_{\mathrm{h}}}{\mathbf{k}_{\mathrm{z}}}
$$

Measurement of the light refractive index in water using a double interferometer

In the experiment with interferometers, while measuring the light refractive index in water, I discovered a new principle for the direct measurement of the light refractive index in water using

a Michelson interferometer as referred to in Figure 3 . The lower half of the rays of light impinges on the shifting mirror in a cuvette with water. The upper half of the rays of light impinges on the mirror in the surrounding atmosphere. The mirror is shifted on the same trajectory $\mathbf{x}$ in the air and in the cuvette water. We can therefore propose the following equation: $\mathbf{k}_{\mathrm{hw}} \lambda_{\mathrm{hw}}=\mathbf{k}_{\mathrm{ha}} \lambda_{\mathrm{ha}}, \frac{\mathbf{k}_{\mathrm{hw}}}{\mathbf{k}_{\mathrm{ha}}}=\frac{\lambda_{\mathrm{ha}}}{\lambda_{\mathrm{hw}}}=\frac{\lambda_{\mathrm{h}}}{\mathbf{n}_{\mathrm{ha}}} \frac{\mathbf{n}_{\mathrm{hw}}}{\lambda_{\mathrm{h}}}=\frac{\mathbf{n}_{\mathrm{hw}}}{\mathbf{n}_{\mathrm{ha}}}, \mathbf{n}_{\mathrm{hw}}=\mathbf{n}_{\mathrm{ha}} \frac{\mathbf{k}_{\mathrm{hw}}}{\mathbf{k}_{\mathrm{ha}}}$

The measured values of the shifts of interference fringes are obtained during the measurement of the light refractive index in water as referred to in Figure 3.

The shifts of the interference fringes are registered by a phototransistor as analogue values of electrical voltage on phototransistor and are converted by AC converters into digital values, which are entered into the computer memory as illustrated on Graph 1.

The graphical display of the shift of the interference fringes in water and in air is calculated using trigonometric functions and can be seen on Graph 2. We can see the high precision of the measurement of the relative light refractive index of water against air.

If instead of one cuvette we use two cuvettes and two mirrors in one arm of the interferometer, the interferometer thus modified will allow us to compare the light refractive index in two different liquids. If we use distilled water or another liquid as a sample liquid which will serve as a reference liquid against which we will compare the concentration of unknown liquid in water, by comparing the light refractive indices of both liquids, we can determine the concentration of the unknown liquid in water with high precision.

Identification of the parameters of light, air and water in a measurement using 


\section{Measurement of the relative index of refraction in the water displacementof the mirror in the water and air}
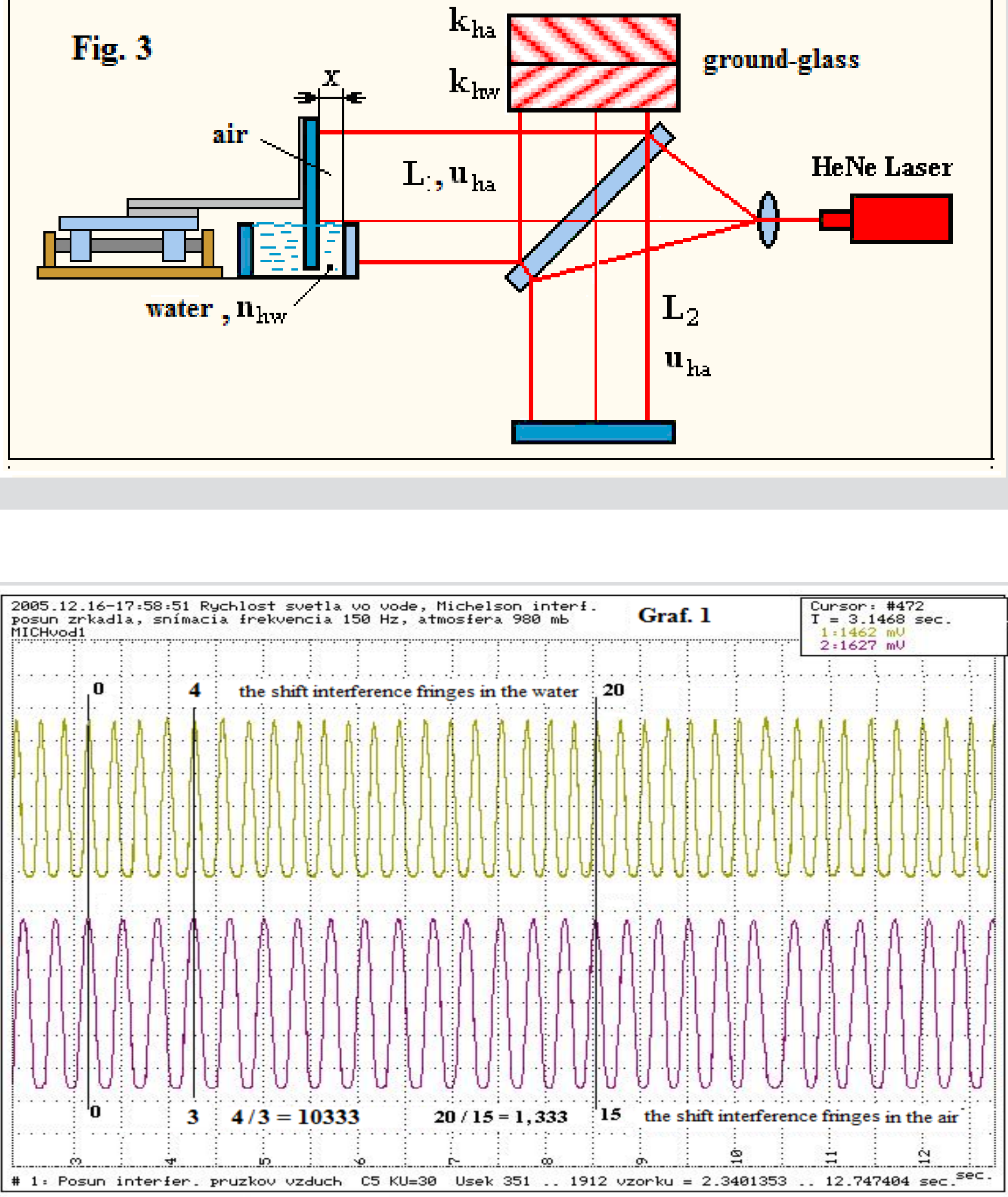


\section{aninterferometer}

The first index will identify the frequency of the light source in the vacuum and the second index will identify the parameters of the air in which the light is propagated. The speed of light is directly proportional to the frequency and wavelength of light $\mathbf{c}=\mathrm{v} \lambda$

"a" Index indicating the light parameters of the air in atmosphere

" $h$ " Index indicating the light parameters of the helium laser in a vacuum

$\mathbf{c}_{\mathrm{h}}=\mathbf{v}_{\mathrm{h}} \lambda_{\mathrm{h}}$ Speed of light of the helium laser in a vacuum

$\mathbf{v}_{\mathrm{h}}$ Frequency of light of the helium laser in a vacuum

$\lambda_{h}$ Wavelength of light of the helium laser in a vacuum

$\mathbf{u}_{\mathrm{ha}}=\mathbf{v}_{\mathrm{ha}} \lambda_{\mathrm{ha}} \quad$ Speed of light of helium laser in air

$\mathbf{v}_{\text {ha }}$ Frequency of light of the helium laser in air

$\lambda_{\text {ha }}$ Wavelength of light of the helium laser in air

$\mathbf{n}_{\text {ha }}$ Absolute light refractive index of the helium laser in the air in atmosphere

$\mathbf{u}_{h w}=\mathbf{v}_{h w} \lambda_{h w}$ Speed of light of the helium laser in water

$\mathbf{v}_{\mathrm{hw}}$ Frequency of light of the helium laser in water

$\lambda_{\mathrm{hw}}$ Wavelength of light of the helium laser in water

$\mathbf{n}_{\text {hw }}$ Absolute light refractive index of the helium laser of air in water

\section{References}

1. http://www.opto.cz/fuka_havelka/t060.html\#TOC1

2. Edlen, B. The refractive index of air. Metrologia 2: $71 \cdot 80 ; 1966$. http://iopscience.iop.org/article/10.1088/0026-1394/30/3/004; http://iopscience. iop.org/article/10.1088/0026-1394/35/2/8

3. W. Sellmeier, https://www.rp-photonics.com/ sellmeier_formula.html

Disclosures: None

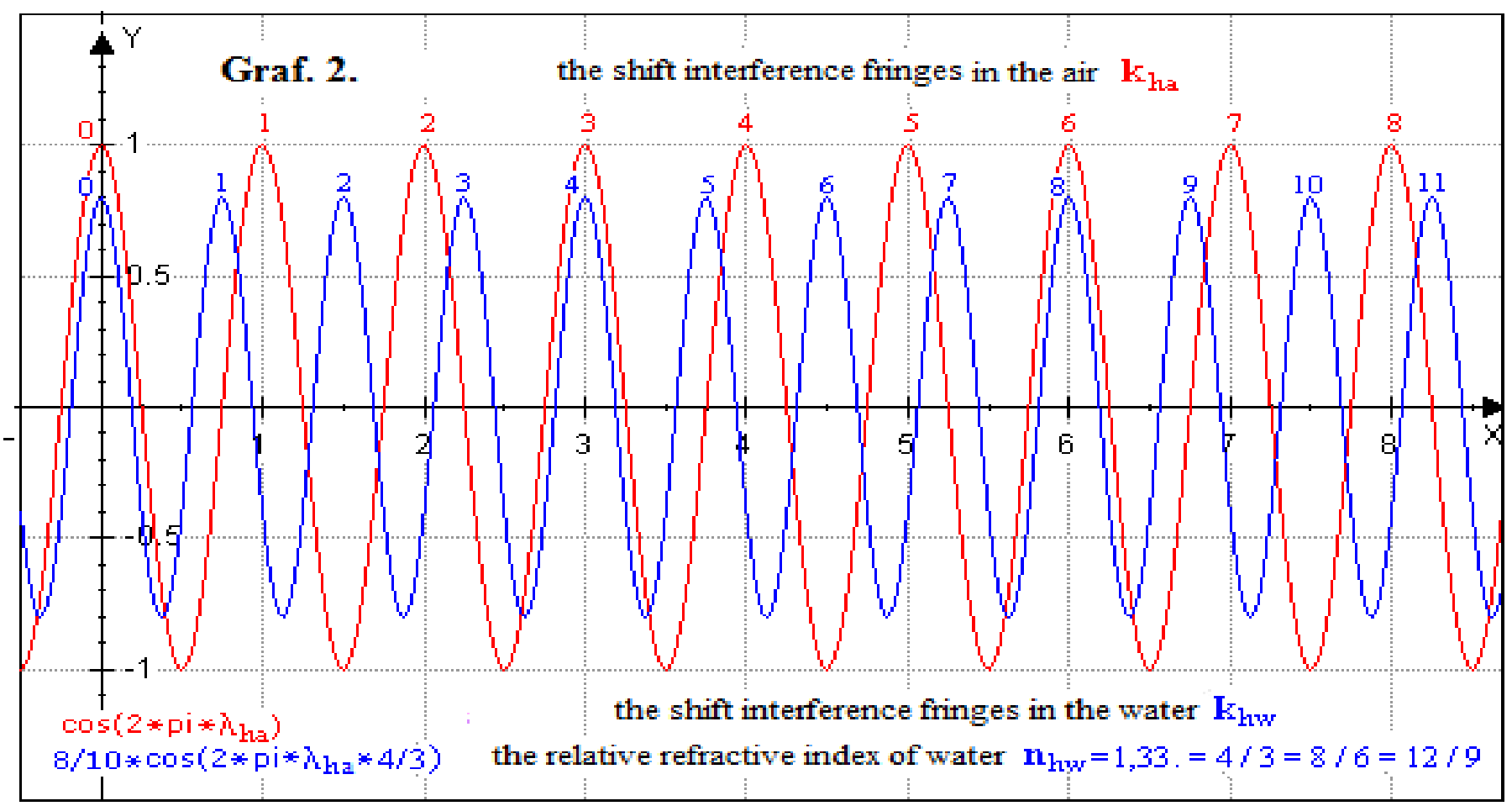

